\title{
Mecanismos De Transporte Del Fluido Dentinario \\ Bases Estructurales
}

\section{Structural Basis Of Dentinal Fluid Carrying Mechanisms}

\author{
Luis H. Gálvez ${ }^{1}$
}

\section{INTRODUCCION}

El tránsito de fluido a través de los túbulos dentinarios, también se puede definir como permeabilidad dentinaria. Para algunos autores solo es posible el flujo dentinario y/o la permeabilidad cuando se pierde el esmalte y el cemento ${ }^{1}$. Sin embargo, existen estructuras adamantinas que facilitan el tránsito de fluidos a través del límite amelodentinario y de todo el espesor del esmalte, como son los husos, penachos, laminillas, estrías de Retzius, etc.

El fluido existente en los túbulos dentinarios, generalmente, es un trasudado pulpar procedente de los vasos pulpares (2), cuyo tránsito suele estar favorecido cuando los túbulos quedan abiertos hacia el exterior por caries, fracturas, preparaciones cavitarias y grabado ácido utilizado en las técnicas adhesivas fundamentalmente.

De manera que, el flujo dentinario está condicionado por las características estructurales de la dentina en condiciones normales y por sus modificaciones en condiciones fisiológicas y patológicas.

\section{MECANISMOS DE TRANSPORTE DE SUSTANCIAS A TRAVÉS DEL TÚBULO DENTINARIO}

Aun cuando la circulación pulpar es normal, existe una pequeña presión hidrostática que se dirige hacia fuera, $y$ a la que se opone el esmalte, el cemento, el barrillo dentinario y las restauraciones. Esta presión disminuye con el empleo de vasoconstrictores en los anestésicos, y aumenta en los procesos inflamatorios; este tipo de transporte puede ocurrir en sentido inverso cuando aumenta la presión exterior, al morder o al realizar restauraciones no refrigeradas, con el consiguiente desprendimiento de calor. (Fig. 1)

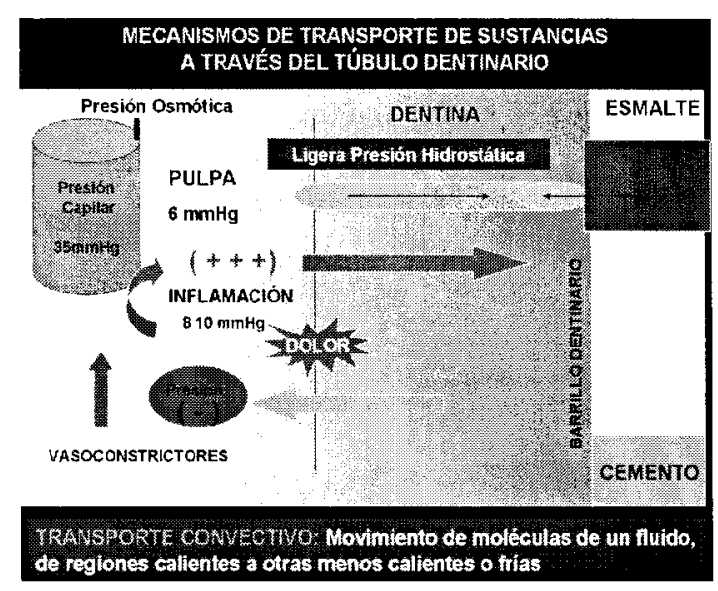

Fig. 1: Transporte Convectivo de adentro afuera

TRANSPORTE CONVECTIVO: Movimiento de moléculas de un fluido, de regiones calientes a otras menos calientes o frías

El Transporte Convectivo se rige por la ecuación de POUESILLE-HAGEN, y es igual a la gradiente de presión durante el movimiento de fluidos por el radio del diámetro tubular, dividido entre la longitud del túbulo y la viscosidad del fluido por la constante de 8. (Fig. 2)

$$
\mathrm{TC}=\mathrm{GPFM} \mathrm{r} 4 / 8 \mathrm{VF}_{\mathrm{F}} \mathrm{LT}_{\mathrm{T}}
$$

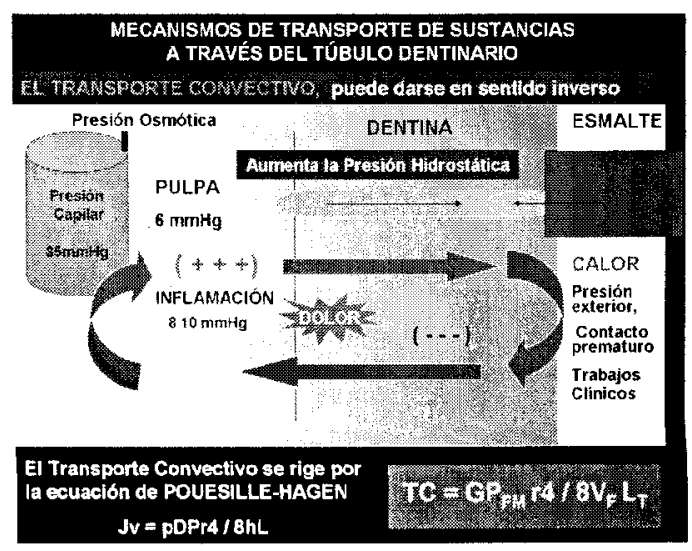

Fig. 2: Transporte Convectivo de afuera adentro 
TRANSPORTE POR DIFUSIÓN: otra posibilidad para el paso de productos a través del túbulo dentinario. De igual manera, se puede correlacionar las variables que intervienen.

El transporte por difusión es igual al coeficiente de difusión multiplicado con la resultante del diámetro tubular por la cantidad de túbulos, por el cambio en la concentración de solutos a través de la dentina dividido entre el espesor de la dentina. (Fig. 3)

$$
\mathrm{TD}=\mathrm{Cd} \mathrm{Sd}{ }^{*} \mathrm{DCs} / \mathrm{De}
$$

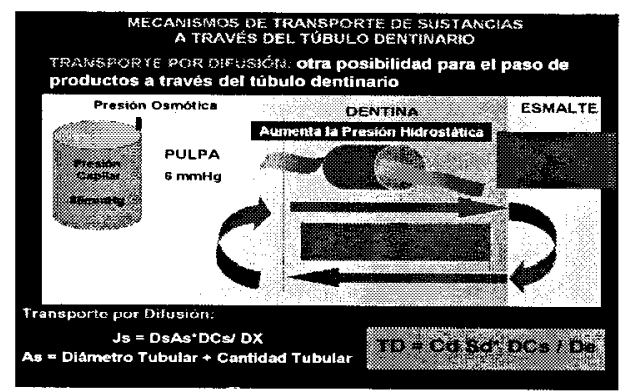

Fig. 3: Transporte por Difusión

TRANSPORTE POR IONTOFORESIS: Los solutos con carga eléctrica pueden verse acelerados cuando se produce una corriente eléctrica, durante las restauraciones metálicas. (Fig. 4)

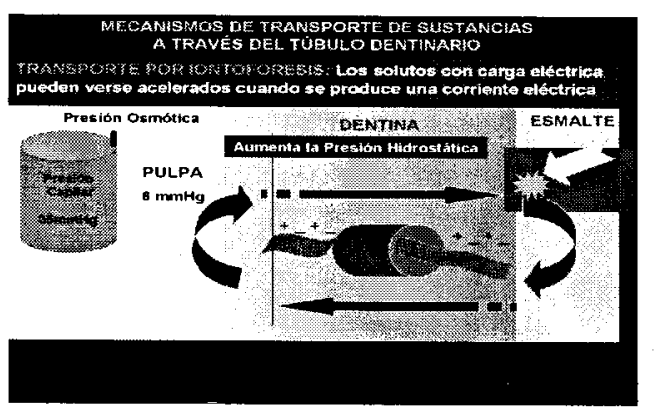

Fig. 4: Transporte por Iontoforesis

\section{BASES ESTRUCTURALES DENTINA}

Tejido de origen mesenquimático, subyacente al esmalte y al cemento dentario, constituye la suma total de matriz extracelular, peritubular e intertubular, producida por la totalidad de dentinoblastos; que se desplaza centripetamente para ubicar su producto en el extremo apical del cuerpo celular y adyacente a sus prolongaciones citoplásmicas: procesos y fibrillas.

\section{MATRIZ DENTINARIA}

La matriz dentinaria extracelular, debido a su naturaleza tubular, es un tejido permeable en toda su extensión, desde la frontera interna, adyacente a la pulpa, a la frontera externa, el límite amelodentinario; $y$ viceversa.

\section{TÚBULOS DENTINARIOS}

Los túbulos dentinarios principales son unos conductos que recorren la totalidad de la dentina desde la cámara pulpar hasta el límite amelodentinario. Contiene: prolongaciones de los dentinoblastos, fibras colágenas, fibras nerviosas amielínicas y un transudado procedente de la pulpa. En ocasiones estas prolongaciones de los dentinoblastos sobrepasan el límite amelodentinario y se introducen en el esmalte constituyendo los husos, que facilitan el paso del fluido dentinario hacia el esmalte.

Los dentinoblastos emiten ramificaciones laterales a intervalos de 1,0 a 2,0 micrones ${ }^{3}$, que contactan con otras prolongaciones de los dentinoblastos adyacentes, lo que condiciona una superficie cribiforme del túbulo y una red de túbulos dentinarios secundarios perpendiculares $u$ oblicuos a los principales, que contactan con los túbulos vecinos ${ }^{4,5}$. Las características morfológicas de los túblulos dentinarios permite el tránsito rápido del fluido, lo que facilita la permeabilidad de la dentina y justifica los síntomas de dolor y sensibilidad ${ }^{6}$.

El número y diámetro de los túbulos dentinarios principales es variable dependiendo de la localización y de la edad del diente. En la dentina próxima al límite amelo-dentinario el número de túbulos es de 15000 por milímetro cuadrado, en el tercio medio 45000 por milímetro cuadrado y en la dentina circumpulpar de 65000 por milímetro cuadrado, cuyos diámetro oscilan entre $0,8 \mu$ en la dentina próxima al límite amelo-dentinario y $2,5 \mu$ de la dentina circumpulpar. ${ }^{7}$. El número de túbulos de la dentina circumpulpar varía según el diente, la edad y la superficie anatómica, siendo menor los túbulos en la superficie mesial y distal (44000-46000 por milímetro cuadrado) que en el resto de las otras tres superficies, en dientes definitivos jóvenes y en primeros premolares y segundos molares, cuando se comparan con terceros molares ${ }^{8}$. En la zona media de la raíz el número de túbulos oscila entre los 32000 y 39000 por milímetro cuadrado y en la región apical entre 8000 y 10000 por milímetro cuadrado, 10

El mayor diámetro y número de los túbulos dentinarios en la dentina circumpulpar, hace que esta sea muy permeable ${ }^{11,12}$. También existen otras zonas de mayor permeabilidad dentinaria, como ocurre en determinadas zonas de nuestros diseños cavitarios, debido a un mayor diámetro y número de los túbulos, como ocurre en los ángulos de las líneas mesiopulpares de las preparaciones de clase I y II y en la superficie axial de las restauraciones de clase $V^{13,14}$. 


\section{Referencias Bibliográficas}

1. RAUSCHENBERGER CR: Permeabilidad Dentinaria. Ramificaciones clínicas. Clin Odont Nort, 1992; 2: 539-555.

2. DRIESSENS FCM, VERBEECK RMH: Biominerals. Boca Raton, CRC Press, 1990; 163-178

3. TEN CATE AR: Histología oral. Desarrollo, estructura y función. 2a Ed. Buenos Aires, Editorial Médica Panamericana, 1986.

4. JONES SJ, BOYDE A: Ultrastructure of dentin and dentinogenesis. En: Linde A (Ed.): Dentin and dentinogenesis. Vol. I. Boca Raton, CRC Press, 1984: 81-134.

5. PASHLEY DH: Dentin: A dynamic substrate -A review. Scan Microsc, 1989; 3: 161-176.

6. MARSHALL GW: Dentina: Microestructura y caracterización. Quintessence (Ed. Esp.), 1995; 8: $160-171$

7. GARBEROGLIO R, BRÄNSTRÖM M: Scanning electron microscopic investigation of human dentinal tubules. Arch Oral Biol, 1976; 21: 355362.

8. SCHLLEMBERG U, KREY G, BOSSHARDT D, NAIR PNR: Numerical density of dentinal tubules at the pulpal wall of human. J. Endodon, 1992; 18: 104-109.
9. WHITTAKER DK, KNEALE MJ: The dentinepredentina interface in human teeth. Br Dent $\mathrm{J}$, 1979; 146: 43-46.

10. CARRIGAN OJ, MORSE DR, FURST ML, SINAI IH: A scanning electron microscopic evaluation of human dentinal tubules acording to age and location. J Endodon, 1984; 10: 359363.

11. PASHLEY DH: Smear layer: Physiological considerations. Oper Dent, 1984; Suppl 3: 1329.

12. FOGEL HM, MARSHALL FJ, PASHLEY DH: Effects of distance from the pulp and thickness on the hydraulic conductance of human reticular dentin. J Dent Res, 1988; 67: 1381-1385.

13. PASHLEY DH, PARSONS GS: Pain produced by topical anesthetic ointment. Endodon Dent Traumatol, 1987; 3: 80.

14. PASHLEY DH: Interactions of dental materials with dentin. Trans Acad Dent Mater, 1990; 3: 55-73. 